\title{
Influencia de factores agroambientales sobre la calidad del clon de cacao (Theobroma cacao L.) PH-16 en la región cacaotera de Bahia, Brasil
}

\section{Influence of agro-environmental factors on the quality of cacao (Theobroma cacao L.) clone $\mathrm{PH}-16$ in the cacao region of Bahia, Brazil}

\author{
Guilherme Amorim Homem de Abreu Loureiro ${ }^{1 *}$, Quintino Reis de Araujo ${ }^{1,2}$, Raul René Valle ${ }^{1,2}$, George \\ Andrade Sodré ${ }^{1,2}$, Soraya Maria Moreira de Souza ${ }^{2}$ \\ ${ }^{1}$ Universidade Estadual de Santa Cruz. Carretera Ilhéus-Itabuna Km 16 s/n. CP. 45662-000, Ilhéus, Bahia, Brasil. \\ ${ }^{2}$ Centro de Pesquisas do Cacau. Comissão Executiva do Plano da Lavoura Cacaueira. Carretera Ilhéus-Itabuna Km $22 \mathrm{~s} / \mathrm{n}$. CP. \\ 45600-970, Itabuna, Bahia, Brasil. \\ *Autor de correspondencia: gahal.85@gmail.com
}

Nota científica recibida: 12 de noviembre de 2016 aceptada: 21 de marzo de 2017

RESUMEN. Los desafíos técnicos y científicos actuales para el cultivo del cacao implican la necesidad de evaluar múltiples atributos relacionados con la producción primaria y con los criterios de calidad. El estudio se realizó con el clon de cacao PH-16 en la zona húmeda de la región cacaotera de Bahia, Brasil. El objetivo fue conocer la influencia del sitio de cultivo sobre las variables físicas y químicas antes y después de la fermentación de los granos de cacao. Las variables químicas más influenciadas por el medio ambiente fueron el $\mathrm{pH}$ y la acidez total de los granos con mucílago, y el $\mathrm{pH}$, acidez total e índice de pigmentos de los granos fermentados. El sitio de cultivo que corresponde al Argisol Amarillo Distrófico latosólico, en el sistema agroforestal Cabruca con densidad media de 35 árboles de sombra por hectárea, presentó los granos con mejores atributos de calidad.

Palabras clave: Beneficio poscosecha del cacao, calidad del cacao, Theobroma cacao L., trazabilidad agrícola

ABSTRACT. Current technical and scientific challenges for cacao cultivation involve the need to evaluate numerous attributes related to primary production and quality criteria. The study was conducted with the cacao clone $\mathrm{PH}-16$, in the humid zone of the cacao region of Bahia, Brazil. The objective was to know the influence of the place of cultivation on the physical and chemical variables, before and after the cacao grains have fermented. The chemical variables that are more liable to be influenced by the environment are the $\mathrm{pH}$ and overall acidity of the grains with mucilage, as well as the $\mathrm{pH}$, overall acidity, and pigment rate of fermented grains. Cultivation areas with latosol dystrophic yellow argisol, in the agro-forestry Cabruca system, with a mean density of 35 shade trees per hectare, had the best grains, quality-wise.

Key words: Cacao post-harvesting processing, cacao quality, Theobroma cacao L., agriculture traceability

\section{INTRODUCCIÓN}

El árbol del cacao (Theobroma cacao L.) se cultiva en 10 millones de hectáreas en los países tropicales con una producción superior de los 4 millones de toneladas (Wood y Lass 2008, FAO 2017). Después del beneficio poscosecha, los granos son el principal producto comercial del cacao, los cuales son la materia prima del chocolate, alimento altamente consumido en el mundo, cuya demanda industrial aumenta cada año (ICCO 2012). Por lo que las inversiones e innovaciones tecnológicas en el cultivo del cacao tienen la intención de aumentar la productividad y la calidad del cacao (Araujo et al. 2014, Badrie et al. 2015).

La estrategia básica para incrementar la productividad del cacao en Brasil, es la multiplicación vegetativa o clonación de genotipos tolerantes a la enfermedad de la escoba de bruja (Monteiro y Ahnert 2012). Pero estos genotipos tienen diferentes 
demandas nutricionales, y son influenciados por la interacción entre el genotipo y el ambiente (Lopes et al. 2011, Muniz et al. 2013). En este contexto, el genotipo Porto Híbrido 16 ( $\mathrm{PH}-16)$, tolerante a la escoba de bruja, se originó a partir de una población de plantas híbridas originadas de la cruza entre genotipos Forasteros y Trinitarios (Cruz et al. 2013). Por lo que es necesario caracterizar sus atributos físicos y químicos de los granos y conocer su calidad (Araujo et al. 2014, Badrie et al. 2015, Loureiro et al. 2016).

Factores ambientales, genéticos y tecnológicos como el manejo poscosecha, la fermentación, el secado y el tostado son fundamentales para la caracterización física y química de los granos de cacao (Afoakwa, 2010). Por lo que es importante controlar el pH, la acidez total, el contenido de sólidos solubles totales del mucílago durante las diferentes etapas de poscosecha. Por lo anterior, objetivo del presente trabajo fue evaluar la influencia de diferentes sitios de cultivo, en las propiedades físicas y químicas de la masa del cacao y de los granos antes y después del beneficio poscosecha.

\section{MATERIALES Y MÉTODOS}

\section{Área de estudio}

Se evaluaron 12 sitios de cultivo de cacao localizados en la región cacaotera del sur de Bahía, Brasil (Tabla 1), zona húmeda con los tipos climáticos de Thornthwaite: B4r A', B3r A', B2r A', B2r $B^{\prime}$, B1r A', B1r' A', B1w A' (SEI 1998). En estos sitios, el clon $\mathrm{PH}-16$ se cultiva bajo los sistemas cacao y caucho [Hevea brasiliensis (Willd. Ex Adr de Juss.)], cacao y erythrina (Erythrina fusca Lour.), y cabruca, con distintas densidades de árboles de sombra por hectárea y diferentes suelos (Tabla 1) de acuerdo con el Sistema Brasileño de Clasificación de Suelos (SiBCS) (Santos et al. 2013).

\section{Muestreo de suelos y frutos del cacao}

En el estudio, el suelo fue el factor que estratificó el sitio (origen) de la muestra y las unidades experimentales fueron los árboles del cacao injertados con el genotipo $\mathrm{PH}-16$, de los que se cosecharon 50 mazorcas maduras dentro del perímetro de un área circular con un radio de aproximadamente $100 \mathrm{~m}$ de distancia entre los puntos de identificación de los suelos por el SiBCS (Santos et al. 2013), en cada sitio se tomaron tres repeticiones al azar. En cada sitio de estudio, una hectárea de cultivo se dividió en tres áreas de cosecha, que se caracterizaron por tener el mismo suelo y sistema de cultivo. Por lo que cada muestra corresponde a un suelo identificado y clasificado.

\section{Fermentación del cacao}

Los granos con mucílago se fermentaron por $168 \mathrm{~h}$ (7 d) en cajas de espuma de poliestireno de $30 \times 20 \times 30 \mathrm{~cm}$ con capacidad aproximada de 8 $\mathrm{kg}$. Después de las $48 \mathrm{~h}$ de fermentación, la masa del cacao se volteó todos los días para oxigenarla y homogeneizarla. Después de la fermentación, los granos se secaron por $192 \mathrm{~h}$ ( $8 \mathrm{~d}$ ), en un horno de ventilación forzada a temperatura de entre 36 y $38{ }^{\circ} \mathrm{C}$. Para después pelarlos de forma manual con una pinza y separar la cáscara de la semilla y el endospermo, el endospermo (cotiledones y embriones) se molió para su posterior análisis químico.

\section{Obtención del extracto del mucílago de los gra- nos}

Se pusieron 20 granos de cacao en un Becker de $50 \mathrm{ml}$, a los que se le adicionaron $200 \mathrm{ml}$ de $\mathrm{H}_{2} \mathrm{O}$ destilada. Para luego homogeneizar y centrifugar por $10 \mathrm{~min}$ a $3000 \mathrm{rpm}$. Para obtener una alícuota de mucílago de cacao de $25 \mathrm{ml}$.

\section{$\mathrm{pH}$ y acidez total}

En el mucílago de los granos y en los endospermos de los granos crudos y fermentados se determinó el potencial de hidrógeno $(\mathrm{pH})$ y la acidez total por los métodos 970.21 y 942.15 de la AOAC (Horwitz y Latimer 2005).

\section{Índice de pigmentos}

El grado de fermentación se estimó en los endospermos de los granos crudos y fermentados por el método del índice de pigmentos (Gourieva y Tserevitinov 1979). 
Tabla 1. Ubicación de los sitios evaluados

\begin{tabular}{|c|c|c|c|c|c|c|c|}
\hline Sitio & $\begin{array}{l}\text { Coordenadas } \\
\text { geográficas }\end{array}$ & $\begin{array}{l}\text { Clasificación } \\
\text { del suelo }{ }^{1}\end{array}$ & $\begin{array}{l}\text { Sigla del } \\
\text { SiBCS }\end{array}$ & $\begin{array}{l}\text { Clasificación } \\
\text { del suelo }\end{array}$ & $\begin{array}{l}\text { Taxonomía } \\
\text { del suelo }\end{array}$ & $\begin{array}{l}\text { Sistema de } \\
\text { Cultivo }\end{array}$ & $\begin{array}{c}\text { Densidad } \\
\text { media de } \\
\text { árboles de } \\
\text { sombra ha } \\
\text { som }\end{array}$ \\
\hline 1 & $\begin{array}{l}13^{\circ} 40^{\prime} 30^{\prime \prime} \mathrm{S}, \\
39^{\circ} 14^{\prime} 27^{\prime \prime} \mathrm{W}\end{array}$ & $\begin{array}{c}\text { Latossolo } \\
\text { Amarelo } \\
\text { Distrófico } \\
\text { Cambissólico }\end{array}$ & LAd cam & $\begin{array}{c}\text { Latosol } \\
\text { Amarillo } \\
\text { Distrófico } \\
\text { cambisólico }\end{array}$ & Hapludox & $\begin{array}{l}\text { Cacao }^{2} \times \\
\text { Caucho }^{3}\end{array}$ & 150 \\
\hline 2 & $\begin{array}{l}13^{\circ} 44^{\prime} 38^{\prime \prime} \mathrm{S} \\
39^{\circ} 30^{\prime} 10^{\prime \prime} \mathrm{W}\end{array}$ & $\begin{array}{c}\text { Argissolo } \\
\text { Vermelho-Amarelo } \\
\text { Distrófico } \\
\text { Típico }\end{array}$ & PVAd & $\begin{array}{l}\text { Argisol } \\
\text { Rojo- } \\
\text { Armarillo } \\
\text { típico }\end{array}$ & Hapludult & $\begin{array}{l}\text { Cacao }^{2} \mathrm{x} \\
\text { Erythrina }^{4}\end{array}$ & 60 \\
\hline 3 & $\begin{array}{l}13^{\circ} 45^{\prime} 21^{\prime \prime} \mathrm{S} \\
39^{\circ} 20^{\prime} 25^{\prime \prime} \mathrm{W}\end{array}$ & $\begin{array}{c}\text { Argissolo } \\
\text { Vermelho-Amarelo } \\
\text { Distrófico } \\
\text { Abrúptico }\end{array}$ & PVAd & $\begin{array}{c}\text { Argisol } \\
\text { Rojo-Amarillo } \\
\text { Distrófico } \\
\text { abrúptico }\end{array}$ & Hapludult & Cabruca $^{5}$ & 60 \\
\hline 4 & 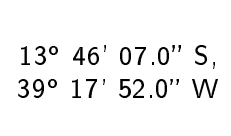 & $\begin{array}{c}\text { Latossolo } \\
\text { Amarelo } \\
\text { Distrófico } \\
\text { Típico }\end{array}$ & LAd & $\begin{array}{l}\text { Latosol } \\
\text { Amarillo } \\
\text { Distrófico } \\
\text { típico }\end{array}$ & $\begin{array}{c}\text { Typic } \\
\text { Hapludox }\end{array}$ & $\begin{array}{l}\text { Cacao }^{2} x \\
\text { Caucho }^{3}\end{array}$ & 350 \\
\hline 5 & $\begin{array}{l}13^{\circ} 51^{\prime} 08^{\prime \prime} \mathrm{S} \\
39^{\circ} 17^{\prime} 54^{\prime \prime} \mathrm{W}\end{array}$ & $\begin{array}{l}\text { Latossolo } \\
\text { Vermelho-Amarelo } \\
\text { Distrófico } \\
\text { Típico }\end{array}$ & LVAd & $\begin{array}{l}\text { Latosol } \\
\text { Rojo-Amarillo } \\
\text { Distrófico } \\
\text { típico }\end{array}$ & $\begin{array}{c}\text { Typic } \\
\text { Hapludox }\end{array}$ & $\begin{array}{l}\text { Cacao }^{2} \times \\
\text { Caucho }^{3}\end{array}$ & 400 \\
\hline 6 & $\begin{array}{lll}14^{\circ} 31^{\prime} & 14^{\prime \prime} \mathrm{S} \\
39^{\circ} & 15^{\prime} & 45^{\prime \prime} \mathrm{W}\end{array}$ & $\begin{array}{c}\text { Argissolo } \\
\text { Vermelho-Amarelo } \\
\text { Eutrófico } \\
\text { Cambissólico }\end{array}$ & PVAe cam & $\begin{array}{c}\text { Argisol } \\
\text { Rojo-Amarillo } \\
\text { Eutrófico } \\
\text { Cambisólico }\end{array}$ & Hapludalf & Cabruca $^{5}$ & 50 \\
\hline 7 & $\begin{array}{l}14^{\circ} 51^{\prime} 36^{\prime \prime} \mathrm{S} \\
39^{\circ} 14^{\prime} 42^{\prime \prime} \mathrm{W}\end{array}$ & $\begin{array}{c}\text { Cambissolo } \\
\text { Háplico } \\
\text { Distrófico } \\
\text { Típico }\end{array}$ & $C X d$ & $\begin{array}{l}\text { Cambisol } \\
\text { Háplico } \\
\text { Distrófico } \\
\text { típico }\end{array}$ & Dystropept & Cabruca $^{5}$ & 35 \\
\hline 8 & $\begin{array}{l}14^{\circ} 51^{\prime} 47^{\prime \prime} \mathrm{S} \\
39^{\circ} 06^{\prime} 47^{\prime \prime} \mathrm{W}\end{array}$ & $\begin{array}{l}\text { Latossolo } \\
\text { Vermelho-Amarelo } \\
\text { Distrófico } \\
\text { argissólico }\end{array}$ & LVAd arg & $\begin{array}{l}\text { Latosol } \\
\text { Rojo-Amarillo } \\
\text { Distrófico } \\
\text { argisólico }\end{array}$ & Hapludox & Cabruca $^{5}$ & 70 \\
\hline 9 & $\begin{array}{lll}15^{\circ} 17^{\prime} & 04^{\prime \prime} \mathrm{S} \\
39^{\circ} & 28^{\prime} & 43^{\prime \prime} \mathrm{W}\end{array}$ & $\begin{array}{l}\text { Argissolo } \\
\text { Amarelo } \\
\text { Distrófico } \\
\text { latossólico }\end{array}$ & PAd lat & $\begin{array}{l}\text { Argisol } \\
\text { Amarillo } \\
\text { Distrófico } \\
\text { latosólico }\end{array}$ & Hapludult & Cabruca $^{5}$ & 35 \\
\hline 10 & $\begin{array}{ll}15^{\circ} 23^{\prime} & 08^{\prime \prime} \mathrm{S} \\
39^{\circ} 26^{\prime} & 04^{\prime \prime} \mathrm{W}\end{array}$ & $\begin{array}{c}\text { Argissolo } \\
\text { Vermelho-Amarelo } \\
\text { Distrófico } \\
\text { Típico }\end{array}$ & PVAd & $\begin{array}{c}\text { Argisol } \\
\text { Rojo-Amarillo } \\
\text { Distrófico } \\
\text { típico }\end{array}$ & Hapludult & Cabruca $^{5}$ & 35 \\
\hline 11 & $\begin{array}{lll}15^{\circ} 23^{\prime} & 15.1^{\prime \prime} \mathrm{S} \\
39^{\circ} & 25^{\prime} & 48.6^{\prime \prime} \mathrm{W}\end{array}$ & $\begin{array}{c}\text { Argissolo } \\
\text { Vermelho-Amarelo } \\
\text { Alítico } \\
\text { Típico }\end{array}$ & PVA ali & $\begin{array}{c}\text { Argisol } \\
\text { Rojo-Amarillo } \\
\text { Alítico } \\
\text { típico }\end{array}$ & Hapludult & Cabruca $^{5}$ & 35 \\
\hline 12 & $\begin{array}{ll}16^{\circ} 29^{\prime} & 02^{\prime \prime} \mathrm{S} \\
39^{\circ} 23^{\prime} & 56^{\prime \prime} \mathrm{W}\end{array}$ & $\begin{array}{c}\text { Argissolo } \\
\text { Vermelho-Amarelo } \\
\text { Distrófico } \\
\text { Coeso }\end{array}$ & PVAd coe & $\begin{array}{c}\text { Argisol } \\
\text { Rojo-Amarillo } \\
\text { Distrófico-cohesivo } \\
\text { abrúptico }\end{array}$ & Hapludult & $\begin{array}{l}\text { Cacao }^{2} \times \\
\text { Caucho }^{3}\end{array}$ & 400 \\
\hline
\end{tabular}

\section{Contenido de sólidos solubles y temperatura}

El contenido de sólidos solubles ( ${ }^{\circ}$ Brix) del mucílago de los granos se determinó con un refrac- tómetro portátil Atago, modelo PAL-1, con corrección directa de la lectura de los grados brix. El control de la temperatura de la masa de cacao 
durante la fermentación se midió en grados Celsius con un termómetro digital, que se colocó a una profundidad de $15 \mathrm{~cm}$.

\section{Análisis estadístico}

Se utilizó un diseño experimental completamente al azar con 12 sitios de estudio caracterizados por diferentes tipos de suelos y sistemas de cultivo con tres repeticiones por sitio. El análisis estadístico se realizó con en el programa $\mathrm{R}$. La prueba de normalidad de Kolmogorov-Smirnov con la corrección de Lilliefors se llevó a cabo con el paquete 'nortest' (Gross y Ligges 2015); mientras que la transformación normalizadora se realizó con la función Normalize del paquete QuantPsyc (Fletcher 2015). El Análisis de Varianza y la prueba de Scott-Knott al 5 $\%$ de probabilidad se realizó con el paquete ExpDes (Ferreira et al. 2015) y los gráficos se realizaron con el paquete Lattice (Sarkar 2016).

\section{RESULTADOS Y DISCUSIÓN}

Por el bajo coeficiente de variación ( $\mathrm{CV}=1.4$ $\%$ ) del pH de la masa de granos de cacao sin fermentar se observa que la variable tuvo variación (Tabla 2). El mucílago del grano de cacao es ácido, con $\mathrm{pH}$ entre 3.0 y 3.5, debido al contenido de ácido cítrico (Penha y Matta 1998). Las reacciones de alta acidez en la fermentación pueden influir en la calidad final de los granos de cacao, lo que compromete el aroma del chocolate (Loureiro et al. 2016). Los valores promedio de $\mathrm{pH}$ de los granos de cacao sin fermentar están dentro del rango reportado por Penha y Matta (1998). Se observan los mayores valores de $\mathrm{pH}$ en los granos de cacao en los sitios de cultivo con suelos 6 - Argisol Rojo-Amarillo Eutrófico cambisólico, 7 - Cambisol Háplico Distrófico típico, 8 - Latosol Rojo-Amarillo Distrófico argisólico y 12 Argisol Rojo-Amarillo Distrófico-cohesivo abrúptico. Lo que indicar que los tipos de suelos donde se cultiva el cacao influye en la acidez de los granos de cacao. Se observó mayor variación de acidez en los granos de cacao sin fermentar que la observada en el $\mathrm{pH}$; la acidificación de los granos permite una serie de reacciones enzimáticas que se asocian con las características finales del chocolate (Schwan y Wheals 2004, Loureiro et al. 2016). El exceso de acidez puede ser perjudicial para la calidad final de los granos de cacao, mientras que menores valores de acidez total se relacionan con la calidad del cacao. Los sitios de cultivo con los menores promedios de acidez total fueron el 9 - Argisol Amarillo Distrófico latosólico $\left(10 \pm 0.69\right.$ meq $\left.\mathrm{NaOH} 100 \mathrm{~g}^{-1}\right)$ y 12 - Argisol Rojo-Amarillo Distrófico-cohesivo abrúptico $\left(7.2 \pm 0.50\right.$ meq $\left.\mathrm{NaOH} 100 \mathrm{~g}^{-1}\right)$. En los granos de cacao fermentado los valores de $\mathrm{pH}$ son aproximadamente dos veces mayor que en los granos de cacao sin fermentar. Mientras que los valores de acidez total de los granos de cacao fermentado son aproximadamente 14 veces menores que la presentada en los granos de cacao sin, lo que indica una reducción considerable de la acidez total en el proceso de fermentación que contribuye a la calidad del cacao.

El contenido de sólidos solubles del extracto del mucílago de los granos de cacao sin fermentar presentó una variación mayor $(\mathrm{CV}=8.1 \%)$ que las observadas en el $\mathrm{pH}$ y la acidez total. La pulpa del cacao se puede clasificar como un producto de alto contenido de acidez $(\mathrm{pH}<4.5)$ y alto contenido de sólidos solubles totales, con valores de alrededor de $18{ }^{\circ}$ brix. Estos dos factores promueven el crecimiento de microorganismos que actúan en la fermentación (Penha y Matta 1998). El contenido de sólidos solubles en el mucílago de los granos cacao es casi cinco veces mayor que el contenido del extracto de la pulpa, con promedio general de 3.83 ${ }^{\circ}$ Brix.

La temperatura máxima de $51{ }^{\circ} \mathrm{C}$ se tuvo en el tercer día de fermentación. Al final del séptimo día de fermentación, la temperatura alcanzó valores mínimos, medios y máximos de 18,20 y $25{ }^{\circ} \mathrm{C}$, respectivamente. Los granos de cacao fermentado no deben tener valores altos de ácidez (Schwan y Wheals 2004, Loureiro et al. 2016), por lo que sobresale el sitio 9 - Argisol Amarillo Distrófico latosólico (7.59 \pm 0.22$)$ con el mayor promedio de $\mathrm{pH}$ en los granos de cacao fermentado (Tabla 3).

La acidez total de los granos de cacao fermentado tuvo valores promedio de 0.88 meq $\mathrm{NaOH}$ 
Tabla 2. Análisis de varianza, prueba de Scott-Knott y análisis descriptivo de los granos de cacao sin fermentar y fermentados del genotipo $\mathrm{PH}-16$.

\begin{tabular}{|c|c|c|c|c|c|c|}
\hline \multirow{5}{*}{$\begin{array}{l}\text { Fuentes de } \\
\text { Variación }\end{array}$} & \multicolumn{3}{|c|}{ Granos sin fermentar } & \multicolumn{3}{|c|}{ Granos fermentados } \\
\hline & \multirow{3}{*}{$\mathrm{pH}$} & Acidez & Contenido de & \multirow{3}{*}{$\mathrm{pH}$} & Acidez & Contenido de \\
\hline & & Total & Sólidos Solubles & & Total & Sólidos Solubles \\
\hline & & $\begin{array}{c}\text { meq } \mathrm{NaOH} \\
100 \mathrm{~g}^{-1}\end{array}$ & ${ }^{\circ}$ Brix & & $\begin{array}{c}\text { meq } \mathrm{NaOH} \\
100 \mathrm{~g}^{-1}\end{array}$ & ${ }^{\circ}$ Brix \\
\hline & \multicolumn{6}{|c|}{ Cuadrados medios } \\
\hline Sitios $^{1}$ & $0.03^{* *}$ & $16.2^{* *}$ & $0.71^{* *}$ & $0.42^{* *}$ & $0.53^{* *}$ & $0.01^{\text {ns }}$ \\
\hline Error & 0.002 & 0.83 & 0.10 & 0.07 & 0.02 & 0.01 \\
\hline CV $(\%)$ & 1.4 & 7.2 & 8.1 & 3.9 & 15.5 & 9.1 \\
\hline Sitios $^{1}$ & \multicolumn{6}{|c|}{ Promedio \pm Desviación Estándar $(n=3)$} \\
\hline 01 LAd cam & $3.29 \pm 0.03^{c}$ & $15.7 \pm 0.59^{a}$ & $3.93 \pm 0.31^{b}$ & $6.65 \pm 0.33^{b}$ & $1.08 \pm 0.24^{c}$ & $1.27 \pm 0.12$ \\
\hline 02 PVAd & $3.26 \pm 0.01^{d}$ & $12.8 \pm 1.17^{c}$ & $3.93 \pm 0.12^{b}$ & $6.64 \pm 0.45^{b}$ & $0.74 \pm 0.19^{d}$ & $1.27 \pm 0.06$ \\
\hline 03 PVAd & $3.40 \pm 0.02^{b}$ & $13.0 \pm 0.23^{c}$ & $3.80 \pm 0.17^{b}$ & $6.72 \pm 0.22^{b}$ & $0.82 \pm 0.15^{c}$ & $1.13 \pm 0.23$ \\
\hline 04 LAd & $3.21 \pm 0.03^{d}$ & $15.8 \pm 1.02^{a}$ & $3.87 \pm 0.21^{b}$ & $6.48 \pm 0.16^{b}$ & $0.94 \pm 0.10^{c}$ & $1.17 \pm 0.06$ \\
\hline 05 LVAd & $3.31 \pm 0.03^{c}$ & $12.1 \pm 0.80^{c}$ & $2.87 \pm 0.25^{c}$ & $6.94 \pm 0.24^{b}$ & $0.64 \pm 0.12^{d}$ & $1.20 \pm 0.10$ \\
\hline 06 PVAe cam & $3.51 \pm 0.02^{a}$ & $13.0 \pm 1.28^{c}$ & $4.47 \pm 0.21^{a}$ & $6.84 \pm 0.25^{b}$ & $0.64 \pm 0.08^{d}$ & $1.27 \pm 0.15$ \\
\hline $07 \mathrm{CXd}$ & $3.51 \pm 0.02^{a}$ & $14.1 \pm 0.35^{b}$ & $4.57 \pm 0.23^{a}$ & $6.84 \pm 0.43^{b}$ & $0.60 \pm 0.14^{d}$ & $1.17 \pm 0.06$ \\
\hline 08 LVAd arg & $3.52 \pm 0.02^{a}$ & $12.5 \pm 1.29^{c}$ & $3.23 \pm 0.12^{c}$ & $6.62 \pm 0.16^{b}$ & $0.46 \pm 0.08^{e}$ & $1.17 \pm 0.06$ \\
\hline 09 PAd lat & $3.43 \pm 0.09^{b}$ & $10.0 \pm 0.69^{d}$ & $3.90 \pm 0.60^{b}$ & $7.59 \pm 0.22^{a}$ & $0.41 \pm 0.05^{e}$ & $1.27 \pm 0.06$ \\
\hline 10 PVAd & $3.37 \pm 0.02^{b}$ & $12.5 \pm 1.14^{c}$ & $4.23 \pm 0.55^{a}$ & $6.03 \pm 0.26^{c}$ & $1.86 \pm 0.14^{a}$ & $1.17 \pm 0.15$ \\
\hline 11 PVA ali & $3.32 \pm 0.03^{c}$ & $11.8 \pm 0.99^{c}$ & $3.80 \pm 0.35^{b}$ & $6.32 \pm 0.04^{c}$ & $1.45 \pm 0.15^{b}$ & $1.23 \pm 0.06$ \\
\hline \multirow[t]{2}{*}{12 PVAd coe } & $3.44 \pm 0.11^{a}$ & $7.2 \pm 0.50^{e}$ & $3.40 \pm 0.17^{c}$ & $6.70 \pm 0.04^{b}$ & $0.87 \pm 0.03^{c}$ & $1.33 \pm 0.21$ \\
\hline & \multicolumn{6}{|c|}{ Muestra General $(n=36)$} \\
\hline Valor mínimo & 3.18 & 6.8 & 2.60 & 5.75 & 0.35 & 1.00 \\
\hline Promedio $\pm \mathrm{DE}$ & $3.38 \pm 0.11$ & $12.5 \pm 2.4$ & $3.83 \pm 0.54$ & $6.69 \pm 0.41$ & $0.88 \pm 0.42$ & $1.22 \pm 0.12$ \\
\hline Valor máximo & 3.55 & 16.9 & 4.70 & 7.76 & 1.98 & 1.50 \\
\hline $\begin{array}{l}\text { }{ }^{1} \text { Sitios de cultivo } \\
\text { Rojo-Amarillo Dis } \\
\text { típico. } 5 \text { LVAd - } \\
\text { - Cambisol Háplic } \\
\text { Distrófico latosóli } \\
\text { PVAd coe - Argis } \\
\text { Desviación estánd }\end{array}$ & $\begin{array}{l}\text { racterizados } \mathrm{p} \\
\text { ffico típico. } 3 \\
\text { osol Rojo-Ama } \\
\text { Distrófico típic } \\
10 \text { PVAd - }\end{array}$ & $\begin{array}{l}\text { tipos de suelo } \\
\text { VAd - Argisol } \\
\text { lo Distrófico tí } \\
8 \text { LVAd arg - } \\
\text { gisol Rojo-Am }\end{array}$ & $\begin{array}{l}1 \text { LAd cam - La } \\
\text { jo-Amarillo Distr } \\
\text { co. } 6 \text { PVAe cam } \\
\text { tosol Rojo-Amar } \\
\text { llo Distrófico. } 1\end{array}$ & $\begin{array}{l}\text { sol Rojo Distr } \\
\text { ico abrúptico. } \\
\text { Argisol Rojo-A }\end{array}$ & $\begin{array}{l}\text { co cambisólicc } \\
\text { LAd - Latosol } \\
\text { rillo Eutrófico } \\
\text { ólico. } 9 \text { PAd l }\end{array}$ & $\begin{array}{l}2 \text { PVAd - Argisol } \\
\text { marillo Distrófico } \\
\text { ambisólico. } 7 \text { Cxd } \\
\text { - Argisol Amarillo } \\
\text { Alítico típico. } 12 \\
\text { de Variación. DE }\end{array}$ \\
\hline
\end{tabular}

$100 \mathrm{~g}^{-1}$, valor que es 14 veces menor que el encontrado en los granos sin fermentar. Esta reducción de la acidez de los granos de cacao durante el proceso de fermentación se ha reportado por Schwan y Wheals (2004). Presentando los menores promedios de acidez los granos de cacao fermentado de los sitios 8 - Latosol Rojo-Amarillo Distrófico argisólico $\left(0.46 \pm 0.08\right.$ meq $\left.\mathrm{NaOH} 100 \mathrm{~g}^{-1}\right)$ y 9 - PAd lat Argisol Amarillo Distrófico latosólico ( $0.41 \pm 0.05$ meq $\mathrm{NaOH} 100 \mathrm{~g}^{-1}$ ). No se observaron diferencias estadísticas entre los promedios del contenido de sólidos solubles de los granos de cacao fermentados, pero el valor promedio de 1.22 es casi tres veces menor que el promedio del extracto de los granos sin fermentar. Lo que se debe a la disminución del contenido de sólidos solubles durante la fermentación debido al consumo de metabolitos primarios, como los azúcares por los microorganismos.
En la fermentación aeróbica, el mucílago que rodea el grano de cacao se degrada por la acción de microorganismos del medio ambiente natural, con la producción de metabolitos tales como etanol y ácidos orgánicos como el acético, cítrico, láctico, y oxálico, subiendo la temperatura hasta los $50{ }^{\circ} \mathrm{C}$ (Schwan y Wheals 2004). Al final del proceso fermentativo muchas reacciones ocurren facilitando la entrada de substancias ácidas en los cotiledones de los granos. Al mismo tiempo algunos ácidos son degradados en compuestos volátiles, que ocasionan que disminuyen la acidez total, además de que se ha reportado una correlación inversa entre $\mathrm{pH}$ y acidez total (Loureiro et al. 2016) de los granos de cacao fermentado (Figura 1a).

El pH y la acidez de los granos son atributos importantes de los genotipos de cacao que sufren alteración durante la fermentación, por lo que no 
Tabla 3. Análisis de varianza, prueba de Scott-Knott y análisis descriptivo de las variables de los granos de cacao crudos y fermentados del genotipo $\mathrm{PH}-16$.

\begin{tabular}{|c|c|c|c|c|c|c|}
\hline \multirow{3}{*}{$\begin{array}{l}\text { Fuentes de } \\
\text { Variación }\end{array}$} & \multicolumn{3}{|c|}{ Granos crudos } & \multicolumn{3}{|c|}{ Granos fermentados } \\
\hline & $\mathrm{pH}$ & $\begin{array}{c}\text { Acidez Total } \\
\text { meq NaOH } \\
100 \mathrm{~g}^{-1}\end{array}$ & $\begin{array}{c}\text { Indice } \\
\text { de } \\
\text { Pigmentos }\end{array}$ & $\mathrm{pH}$ & $\begin{array}{c}\text { Acidez Total } \\
\text { meq } \mathrm{NaOH} \\
100 \mathrm{~g}^{-1}\end{array}$ & $\begin{array}{c}\text { Indice } \\
\text { de } \\
\text { Pigmentos }\end{array}$ \\
\hline & \multicolumn{6}{|c|}{ Cuadrado Medio } \\
\hline Sitios $^{1}$ & $0.06 * *$ & $0.52^{* *}$ & $0.20^{* *}$ & $0.30 * *$ & $3.89 * *$ & $0.18^{* *}$ \\
\hline Error & 0.01 & 0.10 & 0.01 & 0.02 & 0.54 & 0.03 \\
\hline CV $(\%)$ & 2.2 & 5.9 & 10.5 & 2.6 & 10.4 & 20.9 \\
\hline Sitios $^{1}$ & \multicolumn{6}{|c|}{ Promedio \pm Desviación Estándar $(n=3)$} \\
\hline 01 LAd cam & $5.5 \pm 0.2^{b}$ & $5.1 \pm 0.2^{b}$ & $1.0 \pm<0.1^{b}$ & $5.6 \pm 0.2^{c}$ & $7.6 \pm 0.4^{a}$ & $1.0 \pm 0.1^{a}$ \\
\hline 02 PVAd & $5.4 \pm 0.1^{c}$ & $5.4 \pm 0.2^{b}$ & $1.4 \pm 0.1^{a}$ & $5.8 \pm<0.1^{b}$ & $6.1 \pm 1.1^{b}$ & $1.2 \pm 0.2^{a}$ \\
\hline 03 PVAd & $5.5 \pm 0.2^{b}$ & $5.3 \pm 0.2^{b}$ & $0.8 \pm<0.1^{c}$ & $5.6 \pm<0.1^{c}$ & $7.3 \pm 0.2^{a}$ & $1.0 \pm<0.1^{a}$ \\
\hline $04 \mathrm{LAd}$ & $5.2 \pm 0.1^{d}$ & $5.3 \pm 0.2^{b}$ & $0.9 \pm<0.1^{c}$ & $5.4 \pm<0.1^{d}$ & $7.6 \pm 0.5^{a}$ & $1.0 \pm 0.1^{a}$ \\
\hline 05 LVAd & $5.5 \pm 0.1^{b}$ & $5.4 \pm<0.1^{b}$ & $0.9 \pm<0.1^{c}$ & $6.0 \pm 0.2^{b}$ & $6.5 \pm 0.8^{a}$ & $0.8 \pm 0.1^{b}$ \\
\hline 06 PVAe cam & $5.5 \pm<0.1^{b}$ & $6.2 \pm 0.1^{a}$ & $1.4 \pm<0.1^{a}$ & $5.4 \pm 0.1^{d}$ & $7.1 \pm 0.4^{a}$ & $0.6 \pm<0.1^{b}$ \\
\hline $07 \mathrm{CXd}$ & $5.7 \pm<0.1^{a}$ & $5.2 \pm 0.4^{b}$ & $1.0 \pm<0.1^{b}$ & $5.3 \pm 0.1^{d}$ & $7.6 \pm 1.0^{a}$ & $0.6 \pm 0.1^{b}$ \\
\hline 08 LVAd arg & $5.4 \pm 0.2^{c}$ & $5.9 \pm 0.7^{a}$ & $1.1 \pm 0.1^{b}$ & $5.2 \pm 0.2^{d}$ & $8.8 \pm 0.1^{a}$ & $0.4 \pm<0.1^{b}$ \\
\hline 09 PAd lat & $5.7 \pm 0.2^{a}$ & $5.9 \pm 0.2^{a}$ & $1.3 \pm<0.1^{a}$ & $6.3 \pm 0.2^{a}$ & $4.2 \pm 0.5^{c}$ & $0.5 \pm 0.2^{b}$ \\
\hline 10 PVAd & $5.6 \pm<0.1^{b}$ & $5.3 \pm 0.4^{b}$ & $1.5 \pm 0.3^{a}$ & $5.7 \pm<0.1^{b}$ & $6.7 \pm 0.7^{a}$ & $0.8 \pm 0.3^{b}$ \\
\hline 11 PVA ali & $5.7 \pm<0.1^{a}$ & $5.1 \pm 0.1^{b}$ & $0.8 \pm 0.1^{c}$ & $5.7 \pm 0.2^{c}$ & $7.5 \pm 1.3^{a}$ & $0.5 \pm 0.1^{b}$ \\
\hline \multirow[t]{2}{*}{12 PVAd coe } & $5.5 \pm 0.1^{b}$ & $4.6 \pm 0.4^{b}$ & $0.9 \pm 0.1^{b}$ & $5.3 \pm 0.1^{d}$ & $8.0 \pm 0.7^{a}$ & $0.8 \pm 0.3^{b}$ \\
\hline & \multicolumn{6}{|c|}{ Muestra General $(n=36)$} \\
\hline Valor mínimo & 5.2 & 4.4 & 0.7 & 5.0 & 3.8 & 0.3 \\
\hline Promedio $\pm \mathrm{DE}$ & $5.5 \pm 0.2$ & $5.4 \pm 0.5$ & $1.1 \pm 0.3$ & $5.6 \pm 0.3$ & $7.1 \pm 1.3$ & $0.8 \pm 0.3$ \\
\hline Valor máximo & 5.9 & 6.7 & 1.7 & 6.5 & 8.9 & 1.5 \\
\hline
\end{tabular}

${ }^{1}$ Sitios de cultivo caracterizados por tipos de suelos: 1 LAd cam - Latosol Rojo Distrófico cambisólico. 2 PVAd Argisol Rojo-Amarillo Distrófico típico. 3 PVAd - Argisol Rojo-Amarillo Distrófico abrúptico. 4 LAd - Latosol Amarillo Distrófico típico. 5 LVAd - Latosol Rojo-Amarillo Distrófico típico. 6 PVAe cam - Argisol Rojo-Amarillo Eutrófico cambisólico. 7 Cxd - Cambisol Háplico Distrófico típico. 8 LVAd arg - Latosol Rojo-Amarillo Distrófico argisólico. 9 PAd lat - Argisol Amarillo Distrófico latosólico. 10 PVAd - Argisol Rojo-Amarillo Distrófico. 11 PVA ali - Argisol RojoAmarillo Alítico típico. 12 PVAd coe - Argisol Rojo-Amarillo Distrófico-cohesivo abrúptico. GL Grados de Libertad. CV Coeficiente de Variación. DE Desviación estándar. Los niveles de significación para el análisis de la prueba $\mathrm{F}:(* *)$ Error del $1 \%$.

se evalua en los granos crudos (Schwan y Wheals 2004). Después de la fermentación, el endospermo presentó un pH promedio de 5.6 (Tabla 3). El pH es un atributo importante de calidad, debido a que puede indicar exceso de acidez en los granos fermentados, lo que reduce la calidad sensorial (Loureiro et al. 2016). Valores de $\mathrm{pH}$ entre 4.0 y 5.0 pueden reflejar una acidez excesiva, con granos cacao con bajo potencial de sabor y exceso de ácido latico (Lopez 1986). Durante la fermentación, ocurre una disminución del $\mathrm{pH}$, con valores de $\mathrm{pH}$ al final de la fermentación de 5.5 (Wood 1978), considerandose como ideales para los granos de cacao permentados valores de $\mathrm{pH}$ entre 5.0 y 5.5 (Amin et al. 2002). Mientas que el pH óptimo para cacao de calidad debe encontrarse entre 5.0 y 5.4 , lo que indica que $\mathrm{pH}$ menores de 5.0 indican la presencia de ácidos volátiles indeseables para el desarrollo del aroma y sabor del cacao (Loureiro et al. 2016). El pH promedio de granos de cacao fermentados tuvo valores mayores de 5.0, lo que indica que los valores de los granos de cacao evaluados se encuentran en los valores optimos de calidad (Loureiro et al. 2016). Por lo que destacan los valores de $\mathrm{pH}$ de los sitios 9 - Argisol Amarillo Distrófico latosólico $(6.3 \pm 0.2)$ y 2 - Argisol Rojo-Amarillo Distrófico típico (5.8 \pm 0.1$)$.

El índice de pigmentos de los granos es un atributo que evalúa el grado de fermentación a partir del cambio de coloración por la degradación de los fenoles y las antocianinas. La persistencia del color violeta indica una fermentación incompleta de los granos. Valores superiores a 1 indican que los granos no fueron bien fermentados (Gourieva y Tserevitinov 1979). El promedio general del índice de pigmentos de los granos fermentados fue de 0.8 (Tabla 3), 

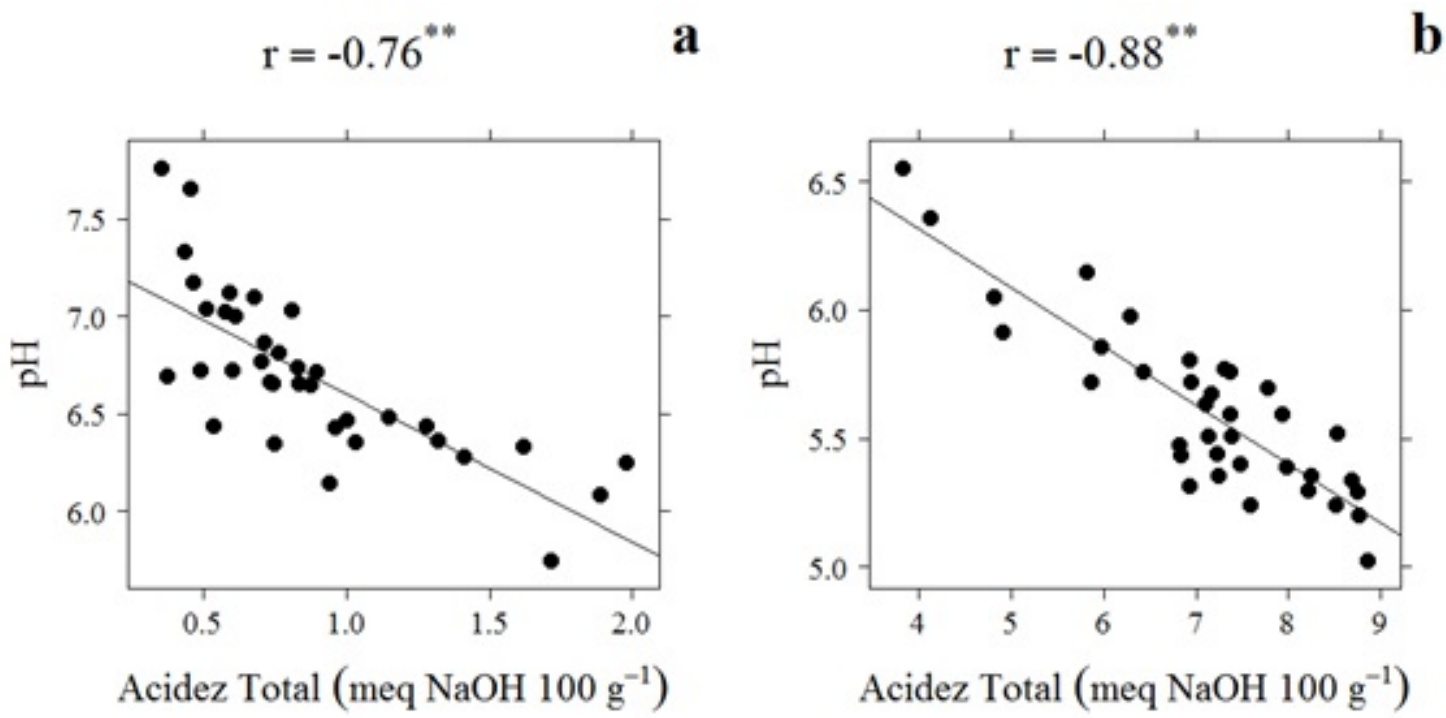

Figura 1. Correlación entre el pH y la acidez total: (a) masa de granos de cacao fermentada; (b) endospermos de los granos del cacao fermentados. Clon de cacao PH-16. $(r=$ Coeficiente de correlación lineal de Pearson; ** Nivel de significación del $1 \%$ de error; $n=36$ ).

por lo que valores menores del índice de pigmentos representan muestras de grano adecuadamente fermentados, como en los sitios: 8 - Latosol RojoAmarillo Distrófico argisólico $(0.4 \pm<0.1), 11$ Argisol Rojo-Amarillo Alítico típico $(0.5 \pm 0.1), 9$ Argisol Amarillo Distrófico latosólico $(0.5 \pm 0.2), 6$ - Argisol Rojo-Amarillo Eutrófico cambisólico (0.6 \pm $<0.1), 7$ - Cambisol Háplico Distrófico típico (0.6 \pm 0.1 ), 5 - Latosol Rojo-Amarillo Distrófico típico (0.8 $\pm 0.1), 10$ - Argisol Rojo-Amarillo Distrófico (0.8 \pm 0.3), 12 - Argisol Rojo-Amarillo Distrófico-cohesivo abrúptico $(0.8 \pm 0.3)$. Se observa que estos sitios están geográficamente localizados al sur con coeficientes de variación del $20.9 \%$, lo que indica que la localización geográfica afecta el índice de pigmentos. Así como el pH, indica la concentración total de ácidos libres (Efraim et al. 2010). La industria del chocolate espera un rango de acidez total entre $12 \mathrm{y}$ 15 meq $\mathrm{NaOH}$ por $100 \mathrm{~g}$ de granos secos (Loureiro et al. 2016). Al respecto se sabe que la acidez total titulada en los granos del cacao aumenta con los días de fermentación (Efraim et al. 2010). Los sitios de cultivo con los menores promedios de acidez total fueron: 2 - Argisol Rojo-Amarillo Distrófico típico $\left(6.1 \pm 1.1\right.$ meq $\left.\mathrm{NaOH} 100 \mathrm{~g}^{-1}\right)$ y 9 - Argisol Amarillo Distrófico latosólico (4.2 $\pm 0.5 \mathrm{meq} \mathrm{NaOH}$ $\left.100 \mathrm{~g}^{-1}\right)$. Pero se observa que los endospermos del genotipo de cacao $\mathrm{PH}-16$ fermentado presentan poca variación en su contenido de acidez total (CV $=10.4 \%$ ) con promedio de 7.1 meq $\mathrm{NaOH} 100$ $\mathrm{g}^{-1}$. Se observaron bajos coeficientes de variación del $\mathrm{pH}$ de los endospermos de los granos crudos y fermentados. La correlación significativa negativa entre el $\mathrm{pH}$ y la acidez total (Figura $1 \mathrm{~b}$ ) de los endospermos de los granos fermentados $(r=-0.88 ; p$ $\leq 0.05$ ), coincide con lo reportado por Efraim et al. (2010) y Loureiro et al. (2016).

Las características químicas que más reflejan la influencia del medio ambiente sobre el beneficio del cacao son el $\mathrm{pH}$ y la acidez total de los granos de cacao, y el pH, acidez total e índice de pigmentos del endospermo de los granos fermentados. El sitio Argisol Amarillo Distrófico latosólico con el sistema Cabruca y densidad media de 35 árboles de sombra por hectárea, presentó las mejores características químicas de calidad de cacao. 


\section{LITERATURA CITADA}

Afoakwa EO (2010) Chocolate science and technology. Chocolate science and technology. Wiley-Blackwell. Oxford. 536p.

Afoakwa EO, Quao J, Takrama J, Budu AS, Saalia FK (2013) Chemical composition and physical quality characteristics of Ghanaian cocoa beans as affected by pulp pre-conditioning and fermentation. Journal of Food Science and Technology 50: 1097-1105.

Amin I, Jinap S, Jamilah B, Harikrisna K, Biehl B (2002) Analysis of vicilin (7S)-class globulin in cocoa cotyledons from various genetic origins. Journal of the Science of Food and Agriculture 82: 728-732.

Araujo QR, Fernandes CAF, Ribeiro DO, Efraim P, Steinmacher D, Lieberei R, et al. (2014) Cocoa Quality Index - a Proposal. Food Control 46: 49-54.

Horwitz W, Latimer GW (2005) Association of Official Analytical Chemists Official Methods of Analysis of the Association of Official Analytical Chemists. 18th ed. AOAC. Gaithersburg, Maryland. 3000p.

Badrie N, Bekele F, Sikora E, Sikora M (2015) Cocoa agronomy, quality, nutritional, and health aspects. Critical Reviews in Food Science and Nutrition 55: 620-659.

Cruz JFM, Bacelar LP, Eduardo SS, Silva BE (2013) Assessment of the fermentative process from different cocoa cultivars produced in Southern Bahia, Brazil. African Journal of Biotechnology 12: 5218-5225.

Efraim P, Pezoa-García NH, Jardim DCP, Nishikawa A, Haddad R, Eberlin MN (2010) Influência da fermentação e secagem de amêndoas de cacau no teor de compostos fenólicos e na aceitação sensorial. Ciência e Tecnologia de Alimentos 30 (Supl.1): 142-150.

FAO (2017) FAOSTAT. Food and agriculture data. Production quantities of cocoa, beans by country. Average 1994-2014. http://www.fao.org/faostat/en/\#data/QC/visualize. Fecha de consulta 16 de marzo de 2017.

Ferreira EB, Cavalcanti PP, Nogueira DA (2015) ExpDes: Experimental designs package. R package version 1.1.2. R: The R Project for Statistical Computing. 42p. http://cran.r-project.org/web/packages/ExpDes/ExpDes.pdf. Fecha de consulta 02 de noviembre de 2016.

Fletcher TD (2015) Package QuantPsyc. R package version 1.5. R: The R Project for Statistical Computing. 26p. http://cran.r-project.org/web/packages/QuantPsyc/QuantPsyc.pdf. Fecha de consulta 02 de noviembre de 2016.

Gourieva MB, Tserevitinov OB (1979) Methods for evaluating the degree of fermentation of cocoa beans. USSR. Patent No. 646.

Gross J, Ligges U (2015) Package "nortest". Tests for normality. R package version 1.0-4. R: The R Project for Statistical Computing. 10p. https://cran.r-project.org/web/packages/nortest/nortest.pdf. Fecha de consulta 02 de noviembre de 2016.

ICCO (2012) International Cocoa Organization. The world cocoa economy. ICCO. London. United Kingdom. 32p.

Lobão DE, Setenta WC, Lobão ESP, Curvelo K, Valle RR (2007) Cacau cabruca - sistema agrossilvicultural tropical. In: Valle RR (ed.) Ciência, tecnologia e manejo do cacaueiro. Gráfica e Editora Vital. Itabuna, Bahia. pp: 290-323.

Lopes UV, Monteiro WR, Pires JL, Clement D, Yamada MM, Gramacho KP (2011) Cacao breeding in Bahia, Brazil - strategies and results. Crop breeding and applied biotechnology S1: 73-81. 
Lopez AS (1986) Chemical changes occurring during the processing of cacao. In Symposium Cacao Biotechnology. University Park. The Pennsylvania State University. pp: 19-54.

Loureiro GAHA, Araujo QR, Sodré GA, Valle RR, Souza Jr JO, Ramos EMLS, et al. (2016) Cacao quality: Highlighting selected attributes. Food Reviews International 33: 382-405.

Monteiro WR, Ahnert D (2012). Melhoramento genético do cacaueiro. En: Valle RR (ed.) Ciência, tecnologia e manejo do cacaueiro. MAPA. Brasília, DF. pp: 1-29.

Muniz MRA, Silveira RLVA, Santos PSR, Malta A, Sorice LSD (2013) Exportação de nutrientes pelos frutos de cacau de diferentes clones cultivados nas Fazendas Reunidas Vale do Juliana. Addubare RR Agroflorestal 24: 5-9.

Penha EM, Matta VM (1998) Caracteristicas fisico-químicas e microbiológicas da polpa de cacau. Pesquisa Agropecuária Brasileira 33: 1945-1949.

Santos HG dos, Jacomine PKT, Anjos LHC dos, Oliveira VÁ de, Lumbreras JF, Coelho MR, et al. (2013). Sistema Brasileiro de Classificação de Solos. $3^{a}$ ed. Embrapa. Brasília, Brasil. 353p.

Sarkar D (2016) Lattice: Multivariate data visualization with R. R package version 0.20-34. R: The R Project for Statistical Computing. 157p. http://cran.at.r-project.org/web/packages/lattice/lattice.pdf. Fecha de consulta 02 de noviembre de 2016.

Schwan RF, Wheals AE (2004) The microbiology of cocoa fermentation and its role in chocolate quality. Critical Reviews in Food Science and Nutrition 44: 205-221.

SEI (1998) Superintendência de estudos Eeconômicos e sociais da Bahia. Tipologia climática - Segundo Thornthwaite - Pluviometria 1943-1983 / Temperatura 1961 - 1990 - Estado da Bahia. http://www.sei.ba.gov.br/site/geoambientais/cartogramas/pdf/carto_tip_clim.pdf. Fecha de consulta 2 de noviembre de 2016.

Wood GAR (1978) Markets for fine flavour versus bulk cocoas. Cocoa Growers' Bulletim 27: 5-11.

Wood GAR, Lass RA (2008) Cocoa. 4th ed. Wiley-Blackwell. Longman. London. 620p. 
